\title{
Feasibility Study on a Measurement Method and a Portable Measuring System to Estimate the Concentration of Cloxacillin and $\beta$-Lactamase in Milk
}

\author{
Luca Ferrari, ${ }^{1}$ Luigi Rovati, ${ }^{2,3}$ Maria Paola Costi, ${ }^{4,5}$ Rosaria Luciani, ${ }^{4,5}$ \\ Alberto Venturelli, ${ }^{4,5}$ and Stefano Cattini ${ }^{2,3}$ \\ ${ }^{1}$ CNH Industrial, Viale delle Nazioni 55, 41122 Modena, Italy \\ ${ }^{2}$ Department of Engineering "E. Ferrari", University of Modena and Reggio Emilia, Via Vivarelli 10, 41125 Modena, Italy \\ ${ }^{3}$ Science e Technology Park for Medicine, TPM, Via 29 Maggio 6, 41037 Mirandola, Italy \\ ${ }^{4}$ Tydock Pharma Srl, Strada Gherbella 294/b, 41126 Modena, Italy \\ ${ }^{5}$ Department of Life Sciences, University of Modena and Reggio Emilia, Via Giuseppe Campi 287, 41125 Modena, Italy
}

Correspondence should be addressed to Alberto Venturelli; a.venturelli@tydockpharma.com and Stefano Cattini; stefano.cattini@unimore.it

Received 27 February 2017; Revised 1 June 2017; Accepted 25 July 2017; Published 30 August 2017

Academic Editor: Nicola Cioffi

Copyright (c) 2017 Luca Ferrari et al. This is an open access article distributed under the Creative Commons Attribution License, which permits unrestricted use, distribution, and reproduction in any medium, provided the original work is properly cited.

\begin{abstract}
Antibiotics are nowadays used and abused worldwide in common veterinary practice to treat diseases, prevent infections, and promote animal's growth. Drug resistance occurrence is a relevant phenomenon that can inactivate the antibiotic. Moreover, antibiotics used for animals are also found in milk and it poses serious health risks to humans. Another negative effect is related to milk processors since antibiotics cause detrimental effects on cheese and yogurt starter bacteria. Given its consumption as both beverage and derivatives, milk is one of the most regulated products in food industry. Nowadays several commercial tests are available to investigate antibiotics in milk, but they generally provide a qualitative result, require bulky procedure, and are time consuming. In this paper, we investigate the use of a chromogenic cephalosporin to quantify the concentration of cloxacillin-a $\beta$-lactam difficult to be detected by using "cowside" screenings which is the drug of choice in the method of mastitis control. The proposed measurement method and prototype have been demonstrated to be able to detect cloxacillin in milk at concentrations lower than the MRL set by the European Commission. Moreover, they are also able to detect the illegal practice of using $\beta$-lactamase to degrade $\beta$-lactams in milk.
\end{abstract}

\section{Introduction}

According to World Health Organization, in 2010, 600 million people suffered foodborne illnesses and 420,000 died [1]. As a result, there is a continuing search for new measurement techniques able to investigate food quality ranging from staple foods such as cereals and cereal products [2-4] to more niche products such as wines $[5,6]$.

Given its consumption as both beverage and derivatives such as cheeses, butter, and yogurt, milk is one of the most regulated products in food industry.

Nowadays almost all livestock receive medication during their lives and antibiotic residues in milk are of great concern to consumers, regulatory agencies, dairy farmers, and milk processors. Indeed, antibiotic residues in milk can [7] (i) seriously spread drug-resistant microorganisms, (ii) cause human health problems such as allergic reaction and imbalance of intestinal microflora, and (iii) badly affect cheese and yogurt starter bacteria. Therefore, there is great interest in methods capable of a fast, reliable, and multiantibiotic sensing.

Among veterinary antibiotics, $\beta$-lactams are one of the most widely used drug classes for the treatment of animals in Europe [8] and all over the world; hence, maximal residue limits (MRLs) have been set by major regulatory agencies, for example, the European Commission Regulation 2377/90. 
Thus, in the last decades, many measurement methods have been developed to detect $\beta$-lactams in food and feed samples $[8,9]$.

The oldest and simpler methods are microbial inhibition tests consisting in adding milk samples to organisms susceptible to the target antibiotics, incubating them, and finally monitoring eventual fails to grow.

Then, many other semiquantitative and quantitative methods such as enzymatic colorimetric assays, microbial receptor assays, receptor binding assays, enzymatic assays, chromatographic analysis, and immunoassays have been developed [7-9]. However, suspected samples detected by qualitative method generally requires being quantified and confirmed by using techniques such as high pressure liquid chromatography (HPLC) and mass spectrometry (MS) [8, 9] which are expensive and impractical for field testing.

Also commercial screening tests such as Charm Farm Cowside by Charm Sciences Inc. (USA), IDEXX SNAP by Thermo Fisher Scientific (USA), Delvotest SP by DSM (Holland), and BetaXpress, TwinsensorBT, and TriSensor by Unisensor Diagnostic Engineering (Belgium) are available to investigate $\beta$-lactams presence in milk samples. Nevertheless, they provide qualitative results only, require bulky procedure, and are time consuming. As an example, the TwinsensorBT requires the following: (i) add $200 \mu \mathrm{l}$ of milk into one reagent microwell and mix, (ii) incubate it for 3 minutes at $40^{\circ} \mathrm{C}$, (iii) dip one Dipstick into each microwell, (iv) continue incubating for $3 \mathrm{~min}$ at $40^{\circ} \mathrm{C}$, and, finally, (v) read the color intensities.

Driven by the continued search for new measurement techniques, several other methods have been proposed (i.e., [10-15]). Nonetheless, most of such methods are laborious and time consuming and require modifications of the antibody used in the assay.

Moreover, sometimes dairy industries illegally add $\beta$ lactamase in milk to degrade $\beta$-lactams antibiotics $[14,16$, 17]. Such illegal practice may reduce $\beta$-lactams concentration, but it exposes consumers to risks to the health since the safety of $\beta$-lactamase has not been fully evaluated [17].

In this paper, we propose a measurement method based on a relatively low cost chromogenic $\beta$-lactamases substrate (CENTA) and we developed and tested a simple and inexpensive prototype measuring instrument potentially able to detect and quantify $\beta$-lactam antibiotics in milk. In particular, this preliminary study has been focused on the possibility of measuring cloxacillin. Among $\beta$-lactam antibiotics, cloxacillin has been chosen since (i) it is from a long time the drug of choice in the method of mastitis control $[7,18]$, (ii) it is difficult to be detected by using multiresidue tests [7], and (iii) its detection limit for some of the most commonly used "cowside" screening tests is above the legal MRLs, resulting in false negatives [7].

Moreover, as it will be discussed in detail, the developed measurement method and system are also intrinsically sensitive to the eventual presence of $\beta$-lactamase illegally added to the milk.

Since cattle produce about $85 \%$ of world milk production (followed by buffaloes with $\approx 11 \%$, goats with $\approx 2 \%$, and sheep with $\approx 1 \%$ ) [19], such preliminary study has been focused on cow's milk.

In the following, Section 2.1 briefly reviews competitive assays at the basis of the proposed measurement method; such method is then described in Section 2.2.

The developed prototype measuring system (proof of concept) and measurement procedure are described in Section 2.3 and the test performed to verify the method and to calibrate and test the measuring system is reported in Section 2.4.

The obtained results are reported and discussed in Section 3, and conclusions are drawn in Section 4.

Finally, detailed descriptions of both the performed data analysis and $\beta$-lactamases detection are reported in Appendices $A$ and $B$, respectively.

\section{Materials and Methods}

2.1. Introduction to Two-Step Competitive Assays and Enzyme Kinetic. As described in detail in Section 2.2, the proposed measurement method is a two-step competitive assay.

Differing from direct assays (noncompetitive), in competitive assays, the concentration/presence of the analyte of interest-the measurand-is measured by adding a known amount of another analyte, competitive with the analyte of interest, then exploiting its ability to compete with analyte added to the sample.

Obviously, the concentration of the competitive analyte added to the solution has to be properly designed to match the required measuring interval for the concentration of the analyte of interest $[20,21]$.

In particular, given the use of a two-step competitive format, the reagent concentration has to be in excess in comparison to the concentration of the measurand.

Then, as it will be described in Section 2.2, the "substrate" is first added to the specimen containing the measurand and, in the second step, the enzyme competitive with the analyte of interest- $\beta$-lactams antibiotics - is added.

For a fully competitive enzyme reaction, the time evolution of the concentration of the enzyme-substrate intermediate complex $[E S](t)$ is [22]

$$
\begin{aligned}
{[E S](t)=} & \frac{\left[E_{0}\right][S]}{K_{S} \cdot\left(1+[I] / K_{I}\right)+[S]} \\
& \cdot\left\{1-e^{-\left[K_{S}\left(1+[I] / K_{1}\right)+\left[S_{0}\right]\right] \cdot k_{1} \cdot t}\right\},
\end{aligned}
$$

where $t$ is the time, $\left[E_{0}\right]$ is the free enzyme concentration at time $t=0,[S](t)$ is the substrate concentration, $\left[S_{0}\right]$ is the substrate concentration at time $t=0,[I](t)$ is the inhibitor concentration, and, $K_{S}, K_{1}$, and $k_{1}$ are constants.

2.2. The Proposed Measurement Method. The proposed measurement method is based on a modification of the competitive binding assay reported by Bebrone et al. [23].

In particular, the method is based on the use of CENTA - a commercially available cephalosporin that can be prepared from the cephalothin [24]-and the $\beta$-lactamase enzyme (AmpC). Indeed, CENTA is not an antibiotic itself; 


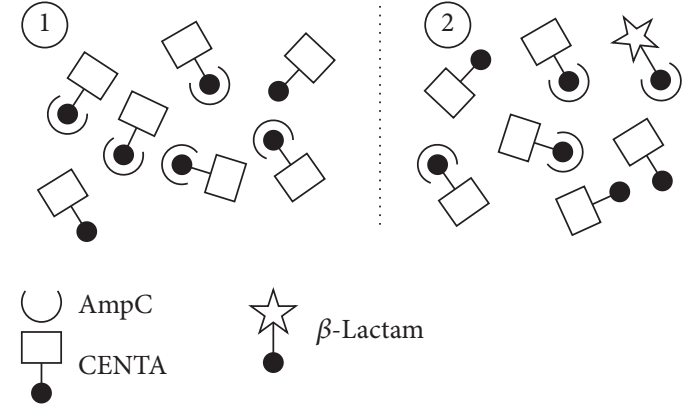

FIGURE 1: Schematic representation of the proposed measurement method. If no antibiotic is present, all the AmpC is linked to the CENTA (1), thus hydrolyzing $\beta$-lactam rings. However, if some $\beta$-lactam antibiotic inhibitor of AmpC is present, some AmpC will link to it, thus reducing the hydrolysis of $\beta$-lactam rings, hence reducing the overall variation of the absorption spectra of the CENTA.

therefore, it can be useful for analytical purpose when the $\beta$-lactam antibiotics have to be detected in a sample matrix by using a competitive assay.

It is known that CENTA is readily hydrolyzed by $\beta$-lactamases (AmpC); thus, according to Jones et al. [25], CENTA reaction with $\beta$-lactamase gives rise to changes in the absorption spectra of the CENTA moving its peak wavelength from about $340 \mathrm{~nm}$ to about $405 \mathrm{~nm}$ concomitantly with hydrolysis of the $\beta$-lactam ring.

According to the results of the inhibition tests reported in Section 3, cloxacillin and other $\beta$-lactams antibiotics are inhibitors of $\beta$-lactamase $(\mathrm{AmpC})$ and reduce their effectiveness. The strength of such inhibition is described by the inhibition constant $\left(K_{i}\right)$ whose value depends on the specific antibiotic and substrate. Therefore, the proposed measurement method is based on the hypothesis that if some $\beta$-lactam antibiotic inhibitor of $\mathrm{AmpC}$ is present in the milk sample, it will compete with the CENTA for the active sites of the $\beta$-lactamase enzymes (AmpC). As a result, we propose a competitive binding assay in two steps in which known amounts of CENTA ([S $\left.S_{0}\right]$ in (1)) and AmpC ([ $\left.E_{0}\right]$ in (1)) are subsequently added to the sample under test, thus analyzing the variation in the absorption spectrum of the sample itself.

According to (1), if no $\beta$-lactams antibiotics are present in the milk sample $([I]=0)$, a fixed and known variation in the absorption spectrum is expected. However, as schematically shown in Figure 1, in the presence of inhibitors, the AmpC is expected to produce a reduced variation of the CENTA spectrum. Therefore, once the concentration of the AmpC $\left(\left[E_{0}\right]\right)$ is fixed, the higher the variation of the absorption spectrum of the CENTA, the lower the concentration of the inhibitor ([I] in (1)) in the tested milk sample.

\subsection{The Developed Prototype Measuring System and Measure-} ment Procedure. According to the enzymes kinetic described in Sections 2.1 and 2.2, the developed measuring system analyzes the time evolution of the absorbance of the sample as a consequence of the additions of known amounts of CENTA and AmpC.

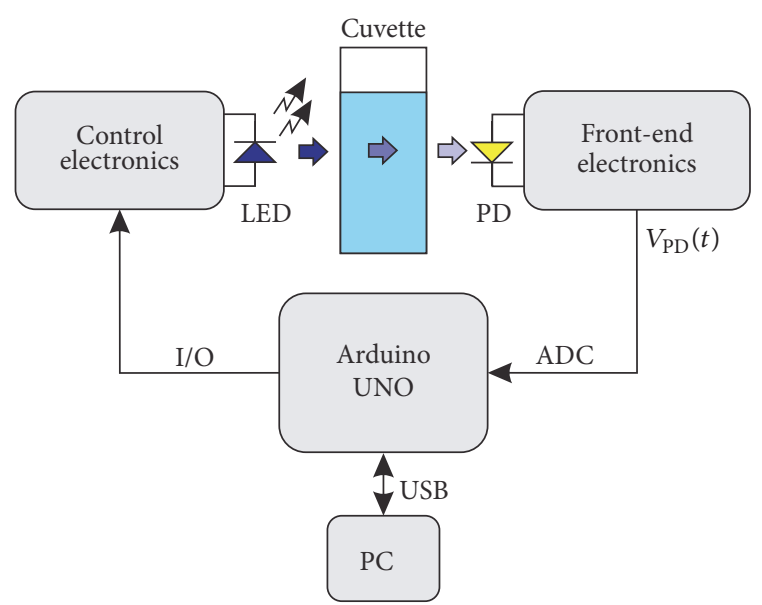

FIGURE 2: Block diagram of the developed prototype measuring system.

TABLE 1: Timing of the proposed measurement procedure. $n$ is the sample index (see Figure 3).

\begin{tabular}{lc}
\hline Interval & Duration \\
\hline$\left[1, n_{1}\right]$ & $50 \mathrm{~s}$ \\
{$\left[n_{1}, n_{2}\right]$} & $\Delta_{t}=15 \mathrm{~s}$ \\
{$\left[n_{2}, n_{3}\right]$} & $225 \mathrm{~s}$ \\
{$\left[n_{3}, n_{4}\right]$} & $\Delta_{t}=15 \mathrm{~s}$ \\
{$\left[n_{4}, n_{\mathrm{TOT}}\right]$} & $280 \mathrm{~s}$ \\
\hline
\end{tabular}

In particular, as shown in Figure 2 during measurement the milk sample is placed in a cuvette with an optical path of $1 \mathrm{~mm}$; hence, it is illuminated by using a LED with a peak wavelength of $405 \mathrm{~nm}$ (model, LED-405-33V, Roithner LaserTechnik-nominal full width at half maximum at $20 \mathrm{~mA}$ equal to $15 \mathrm{~nm}$ ) and the absorbance at such wavelength is estimated by using the photodiode PD (model HFD 1100, EG\&G).

The system is then controlled by using an Arduino UNO board which (i) switches on/off the LED, (ii) analyzes the signal provided by the photodiode, and (iii) communicates with the PC through a USB port. Indeed, the control electronic is basically composed of a constant current generator, whereas the front-end electronic is composed of a transimpedance amplifier and an instrumentation amplifier. The output voltage $V_{\mathrm{PD}}(t)$ of the front-end electronic is digitalized by using a 10-bit ADC included in the Arduino UNO board.

The so acquired data are transmitted to the PC and elaborated according to the measurement model described in the following.

In particular, the system analyzes the acquired $V_{\mathrm{PD}}(n)$ digitized samples of $V_{\mathrm{PD}}(t)$ signal according to the timing diagram shown in Figure 3 and described in Table 1. Supposing that the measurement starts at time $t=t_{0}$ and then adding the CENTA and the AmpC at times $t_{\text {CENTA }}$ and $t_{\text {AmpC }}$, respectively, $V_{\text {Milk }}$ and $V_{\text {CENTA }}$ values are the mean and $V_{\mathrm{PD}}(i)$ 


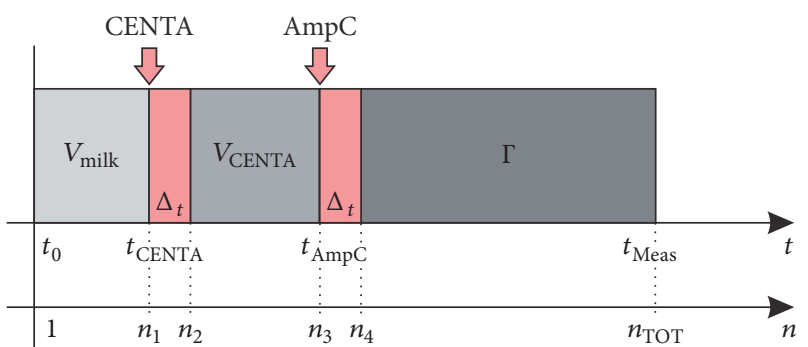

FIGURE 3: Timing diagram of the developed measurement procedure. Supposing that the measurement starts at time $t=t_{0}$ and stops at time $t=t_{\text {Meas }}$, the CENTA and the AmpC were added manually to the sample under test at times $t_{\mathrm{CENTA}}$ and $t_{\mathrm{AmpC}}$ by Gilson pipettes. $\Delta_{t}$ is time intervals after addition of CENTA and AmpC. $n$ is the sample index.

values recorded during the time intervals $\left[t_{0}, t_{\text {CENTA }}\right)$ and $\left[t_{\mathrm{CENTA}}+\Delta_{t}, t_{\mathrm{AmpC}}\right)$, respectively:

$$
\begin{aligned}
V_{\text {Milk }} & =\frac{1}{n_{\text {Milk }}} \cdot \sum_{n=1}^{n_{1}-1} V_{\mathrm{PD}}(n) \\
V_{\mathrm{CENTA}} & =\frac{1}{n_{\mathrm{CENTA}}} \cdot \sum_{n=n_{2}}^{n_{3}-1} V_{\mathrm{PD}}(n) .
\end{aligned}
$$

Then, as described in Appendix A, the antibiotic concentration $\left[C_{\beta}\right]$ has been estimated by analyzing the final or steadystate pseudo-absorbance $\Gamma_{\infty}$ :

$$
\left[C_{\beta}\right]=\frac{a}{\Gamma_{\infty}}-b
$$

where $a$ and $b$ are the constants of the calibration function and, as described in Appendix $A, \Gamma_{\infty}$ was estimated by interpolating the $\Gamma$ function defined as

$$
\Gamma\left(n-n_{4}\right) \mid n \in\left[n_{4}, n_{\mathrm{TOT}}\right]=\log _{10} \frac{V_{\mathrm{CENTA}}-V_{\text {Dark }}}{V_{\mathrm{PD}}(n)-V_{\text {Dark }}},
$$

with the function

$$
\Gamma(i)=\Gamma_{\infty}-\beta \cdot e^{-\alpha\left(i+i_{x}\right)},
$$

where $i$ was the independent variable, $\Gamma(i)$ was the dependent variable, and $\Gamma_{\infty}, \beta, \alpha$, and $i_{x}$ were the parameters.

As described in Appendix A, $V_{\text {Dark }}$ value in (4) is the mean $V_{\mathrm{PD}}$ value recorded before starting the measurement and replacing the cuvette with a beam blocker. Such value was exploited to compensate offsets.

Similarly, $V_{\text {Milk }}$ value defined in (2) was exploited to check for anomalies due, as an example, to variation in the power emitted by the LED and/or unnatural absorbance of the milk sample. Moreover, as described in Appendix B, $V_{\text {Milk }}$ also allows potentially detecting the illegal practice of adding $\beta$-lactamase to milk.

A picture of the developed prototype measuring system is shown in Figure 4.

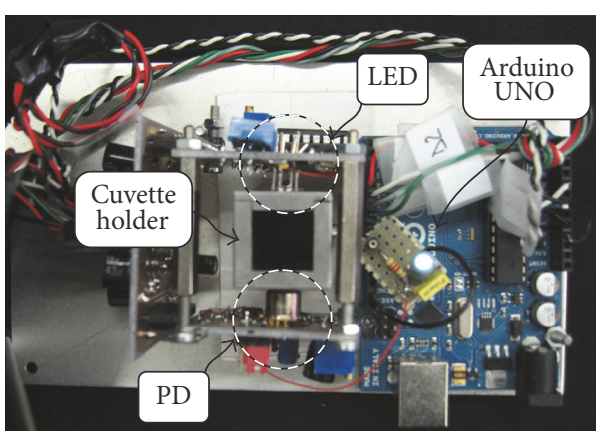

FIgURE 4: Picture of the developed prototype measuring system. In the figure, it is possible to see the LED, the photodiode PD, the cuvette holder, and the Arduino UNO board. During measurements, the system was enclosed in a plastic box to minimize the effects due to environmental lighting and stray-light.

2.4. The Performed Experimental Activities. As previously introduced, the performed experimental activities have been aimed at investigating both the feasibility of the proposed measurement method and the performance of the developed prototype measuring system.

Thus, four different activities were performed: (i) inhibition test, (ii) feasibility verification, (iii) system calibration, and (iv) system verification.

All experiments have been performed at room temperature and without controlling/modifying the temperature of the milk samples.

All the used milk was whole-milk for human consumption and it was bought on the market; thus, it was supposed to be free of antibiotics. Measurements have been then obtained without diluting the milk samples.

Finally, an Escherichia coli K12 AmpC $\beta$-lactamase [26] was used for all experimental activities. The concentration of AmpC was determined spectrophotometrically in concentrated stock solutions made from lyophilized powder and subsequently diluted; this enzyme had been previously expressed and purified, as described by Powers et al. [27].

2.4.1. Inhibition Test. Since the basic idea of the proposed method is that $\beta$-lactams could behave as inhibitors of AmpC-type $\beta$-lactamases, the strength of such inhibition has been evaluated in terms of inhibition constant $\left(K_{i}\right)$ towards AmpC $\beta$-lactamase in buffer and CENTA substrate. In particular, besides cloxacillin, the test investigated cefalexin, cefoperazone, cefalonium, oxacillin, and dicloxacillin.

2.4.2. Feasibility Verification. Feasibility tests have been aimed at investigating the stability of CENTA and AmpC in the milk matrix. Indeed, in case of reagents instability, the overall signal would be decreased for the concentration decrease and not for the enzymatic reaction.

All the feasibility tests have been performed by using an UV/Vis Spectrophotometers (Lambda 25, Perkin Elmerscan-rate set equal to $60 \mathrm{~nm} / \mathrm{min}$ ) and a glass cuvette with an optical path of $1 \mathrm{~mm}$. Then, all measurements have been performed at room temperature and without incubating 
TABLE 2: Performed feasibility tests and relative component concentrations. ${ }^{*}$ Buffer $\left(\mathrm{K}_{x} \mathrm{H}_{y} \mathrm{PO}_{4}, \mathrm{pH} 7.00\right)$ volume added to reach the final cuvette volume of $300 \mu \mathrm{l}$. ${ }^{* *}$ Milk volume added to reach the final cuvette volume of $300 \mu \mathrm{l}$.

\begin{tabular}{lcc}
\hline Component & $\begin{array}{c}\text { Stock } \\
\text { concentration }\end{array}$ & $\begin{array}{c}\text { Cuvette } \\
\text { concentration }\end{array}$ \\
\hline $\begin{array}{l}\text { FT1, buffer } \\
\text { Buffer }\end{array}$ & & \\
CENTA & $5 \mathrm{mM}$ & $240 \mu \mathrm{M}$ \\
AmpC & $13.85 \mu \mathrm{M}$ & $130 \mathrm{nM}$ \\
\hline FT2, milk & & \\
Milk* & \\
CENTA & $5 \mathrm{mM}$ & $240 \mu \mathrm{M}$ \\
AmpC & $13.85 \mu \mathrm{M}$ & $130 \mathrm{nM}$ \\
\hline FT3, antibiotic & & \\
Milk* & \\
CENTA & & $240 \mu \mathrm{M}$ \\
AmpC & $5 \mathrm{mM}$ & $130 \mathrm{nM}$ \\
Cloxacillin & $13.85 \mu \mathrm{M}$ & $5 \mathrm{nM}$ \\
& $0.5 \mu \mathrm{M}$ & $10 \mathrm{nM}$ \\
\hline
\end{tabular}

the samples. As resumed in Table 2, the feasibility test " 1 " (FT1) has been aimed at acquiring the "standard" spectra of CENTA, thus firstly measuring the absorbance of buffer and then the absorbance of buffer and CENTA and finally measuring time evolution of the absorbance of the sample composed of buffer, CENTA, and AmpC after the addition of AmpC (4 spectra, $t \in[0,25]$ minutes). The second feasibility test (FT2) has been aimed at investigating the effect of the milk on the absorption spectra of the CENTA. Hence, FT2 has been performed by firstly measuring the absorbance of the milk, then obtaining the absorbance by adding CENTA, and finally measuring the time evolution of absorbance resulting from the addition of the AmpC (7 spectra, $t \in[0,25]$ minutes). The third feasibility test (FT3) has been aimed at investigating the effects of the competition between $\beta$-lactam antibiotics and CENTA on the optical absorbance of milk samples. Therefore, as reported in Table 2, the test FT3 investigated the spectra obtained from 4 samples: (i) milk (zero concentration of antibiotic), (ii) milk (zero concentration of antibiotic), CENTA, and AmpC, (iii) milk and antibiotic (cloxacillin, concentration $5 \mathrm{nM}$ ), CENTA, and AmpC, and (iv) milk and antibiotic (cloxacillin, concentration $10 \mathrm{nM}$ ), CENTA, and AmpC.

Note that the maximal residue limit (MRL) defined by the European Medicines Agency for cloxacillin in milk is about $70 \mathrm{nM}$ [7]; therefore, about an order of magnitude is greater than the selected concentration of cloxacillin in our sample (see Table 2) (cloxacillin molecular weight is $435.88 \mathrm{~g} / \mathrm{mol}$ ). As a result, the FT3 tests were aimed also at a rough estimate of the potential "detection limit."

2.4.3. Calibration. Calibration activities have been aimed at investigating the relation between the antibiotic concentration $\left(\left[C_{\beta}\right]\right)$ and the pseudo-absorbance $\Gamma_{\infty}$. In particular, as reported in Table 3 , the calibration of the measuring system
TABLE 3: Nominal concentrations of the standards realized for calibration and verification. ${ }^{*}$ Milk volume $80 \mu \mathrm{l} .{ }^{* *}$ Buffer $\left(\mathrm{K}_{x} \mathrm{H}_{y} \mathrm{PO}_{4}\right.$, $\mathrm{pH}$ 7.00) volume added to reach the final cuvette volume of $800 \mu \mathrm{l}$.

\begin{tabular}{lcc}
\hline Component & $\begin{array}{c}\text { Stock } \\
\text { concentration }\end{array}$ & $\begin{array}{c}\text { Cuvette } \\
\text { concentration }\end{array}$ \\
\hline Milk $^{*}$ & & \\
Buffer $^{* *}$ & & \\
CENTA & $8 \mathrm{mM}$ & $261 \mu \mathrm{M}$ \\
AmpC & $13.85 \mu \mathrm{M}$ & $165 \mathrm{nM}$ \\
& & $35 \mathrm{nM}$ \\
& & $70 \mathrm{nM}$ \\
Cloxacillin & $50 \mu \mathrm{M}$ & $105 \mathrm{nM}$ \\
& & $140 \mathrm{nM}$ \\
& & $280 \mathrm{nM}$ \\
& & $420 \mathrm{nM}$ \\
\end{tabular}

TABLE 4: Results of the inhibition test: inhibition constant $\left(K_{i}\right)$ of $6 \beta$-lactam antibiotics towards AmpC $\beta$-lactamase in CENTA substrate.

\begin{tabular}{lc}
\hline Antibiotic & $K_{i}$ \\
\hline Dicloxacillin sodium salt hydrate & $0.85 \pm 20 \% \mathrm{nM}$ \\
Cloxacillin sodium monohydrate & $1.37 \pm 20 \% \mathrm{nM}$ \\
Oxacillin sodium monohydrate & $3.73 \pm 20 \% \mathrm{nM}$ \\
Cefalexin & $17.1 \pm 20 \% \mu \mathrm{M}$ \\
Cefalonium hydrate & $82.6 \pm 20 \% \mu \mathrm{M}$ \\
Cefoperazone dehydrate & $166 \pm 20 \% \mu \mathrm{M}$ \\
\hline
\end{tabular}

had been performed by realizing 8 measurement standards based on milk and antibiotic at known concentrations.

Then, each standard was analyzed according to the measurement procedure described in Section 2.3.

2.4.4. Verification. Verification has been performed by analyzing two (nominally equal) sets of milk samples with known antibiotic concentration, thus comparing the known concentration with results provided by the calibrated system. The composition of the used milk samples is reported in Table 3.

\section{Results and Discussion}

3.1. Inhibition Test. Inhibition test is resumed in Table 4 . According to the reported results, the best inhibitors and thus the easier detectable antibiotics are dicloxacillin, cloxacillin, and oxacillin.

3.2. Feasibility Verification. The results of feasibility tests are shown in Figures 5-8.

As expected and shown in Figure 5, the CENTA spectrum presents an absorption peak at $345 \mathrm{~nm}$. In the presence of the AmpC, the CENTA reacts with the AmpC active site and the absorption peak is shifted to $405 \mathrm{~nm}$.

As shown in Figure 6, the presence of milk predictably modifies the absorption spectra with respect to the spectra 


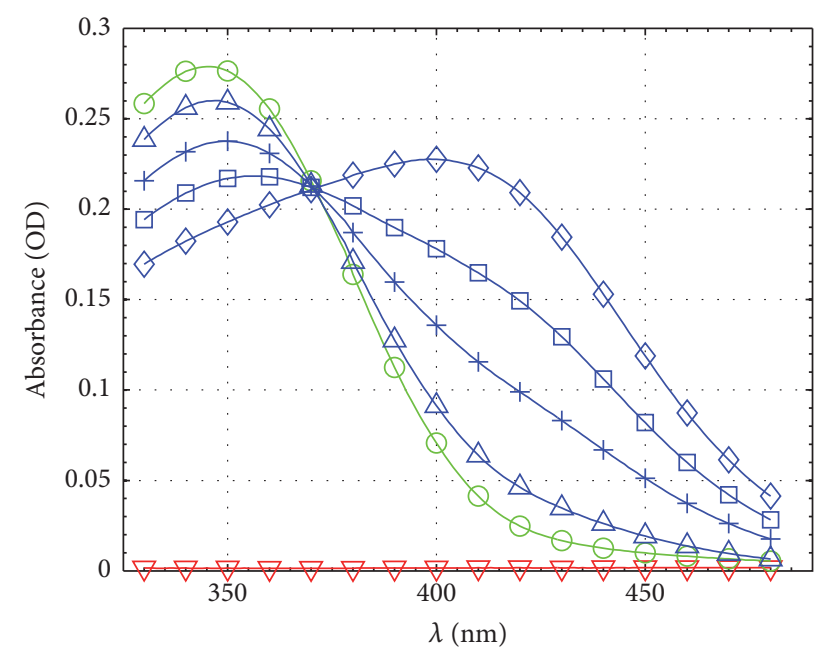

FIGURE 5: Absorbance curves of the buffer solution $(\nabla)$, buffer solution and CENTA $(\mathrm{O})$, and buffer solution, CENTA, and AmpC at consecutive time intervals (curves $\triangle,+, \square, \diamond$ ) (Test PT1; see Table 2).

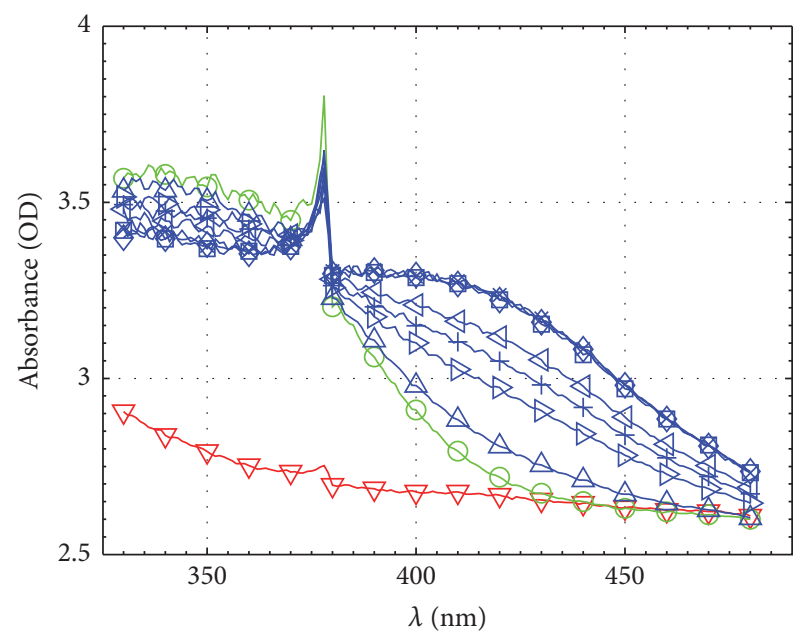

FIGURE 6: Absorbance curves of milk $(\nabla)$, milk and CENTA $(\bigcirc)$, and milk, CENTA, and AmpC at consecutive time intervals (curves $\triangle, \triangleright,+, \triangleleft, \square, \times, \diamond)($ Test PT2; see Table 2).

recorded in buffer solution (mainly the signal to noise ratio). Nevertheless, the AmpC is still able to modify the spectra. Note that the spikes in Figure 6 at about $380 \mathrm{~nm}$ are reasonably measurement artifacts due to the lamp change performed by the spectrometer at such wavelength.

Finally, the settled absorbance spectra obtained by samples with and without antibiotics (FT3) are shown in Figure 7. As expected, the antibiotic competes with the CENTA for the AmpC protein active site; thus, the same amount of AmpC gives rise to a reduced variation of the absorption spectrum (see Figure 1). Such reduction in the effect of AmpC is visible also in Figure 8 where the time evolution of the samples absorbance at $405 \mathrm{~nm}$ is shown. Once again, the spikes at

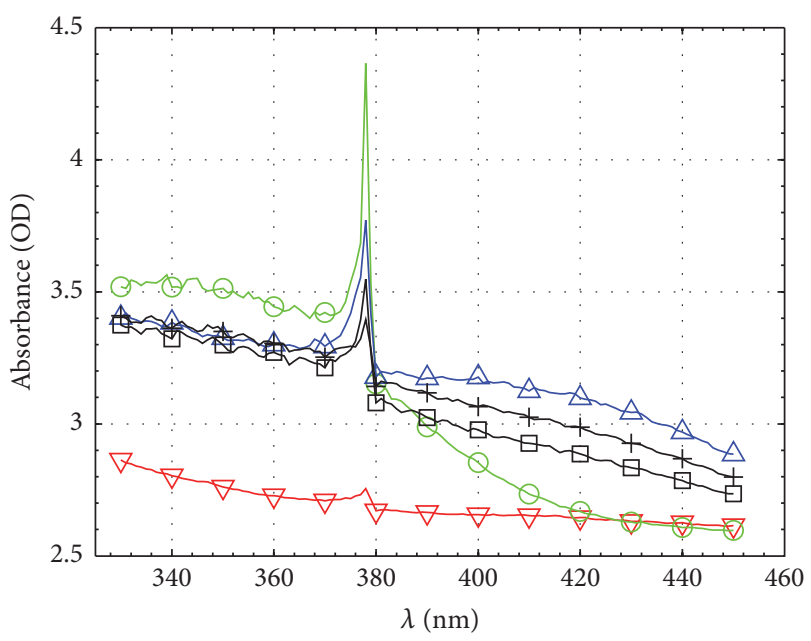

FIGURE 7: Absorbance settled curves of milk $(\nabla)$, milk and CENTA $(O)$, milk, CENTA, and AmpC $(\triangle)$, milk, CENTA, AmpC, and antibiotic $(5 \mathrm{nM})(+)$, and milk, CENTA, AmpC, and antibiotic (10 nM) (ם) (PT3; see Table 2).

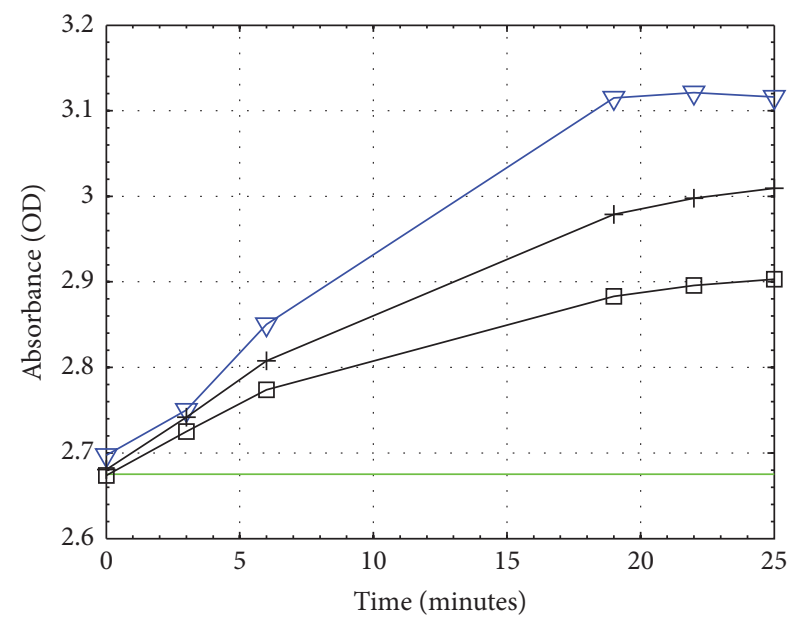

FIGURE 8: Time evolution of the absorbance recorded at $405 \mathrm{~nm}$ for the samples composed of milk, CENTA, and AmpC. In particular, $(\nabla)$ is the absorbance recorded without antibiotic, $(+)$ is the absorbance recorded with antibiotic concentration equal to $5 \mathrm{nM}$, and $(\square)$ is the absorbance recorded with antibiotic concentration equal to $10 \mathrm{nM}$ (PT3, see Table 2). The horizontal line (-) represents the absorbance recorder at $405 \mathrm{~nm}$ at the beginning of the test for a sample composed of milk and CENTA with CENTA concentration equal to $240 \mu \mathrm{M}$.

about $380 \mathrm{~nm}$ in Figure 7 are reasonably measurement artifacts due to the lamp change performed by the spectrometer at such wavelength.

3.3. Calibration. Calibration has been performed according to the procedure described in Section 2.4. An example of $V_{\mathrm{PD}}(n)$ signal recorded using the developed measuring system is shown Figure 9. The results obtained by the analysis of the standards described in Table 3 are shown in Figure 10.

The calibration function has been obtained by least square fitting. 


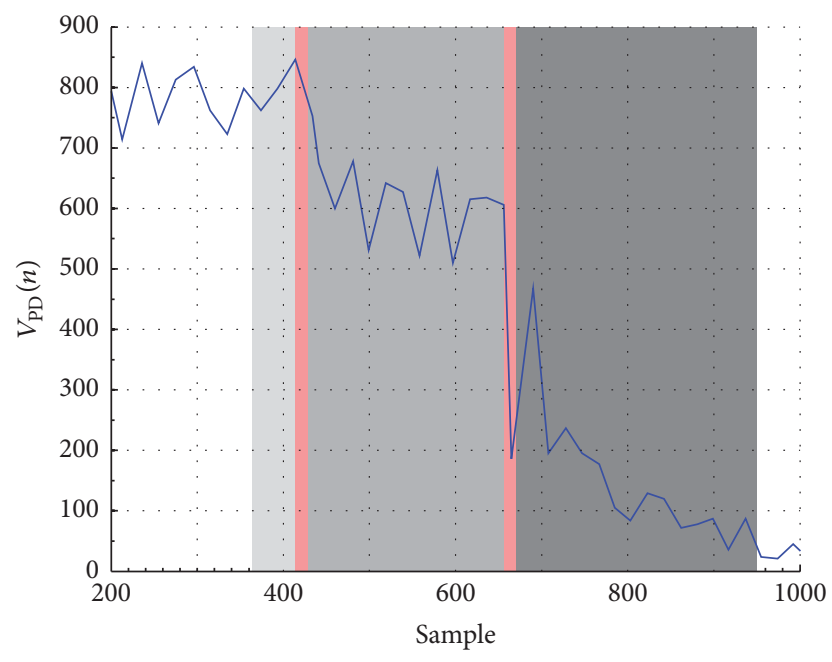

Figure 9: Example of $V_{\mathrm{PD}}(n)$ signal recorded using the developed prototype measuring system. The colored rectangles in the background of the figure refer to the timing diagram shown in Figure 3.

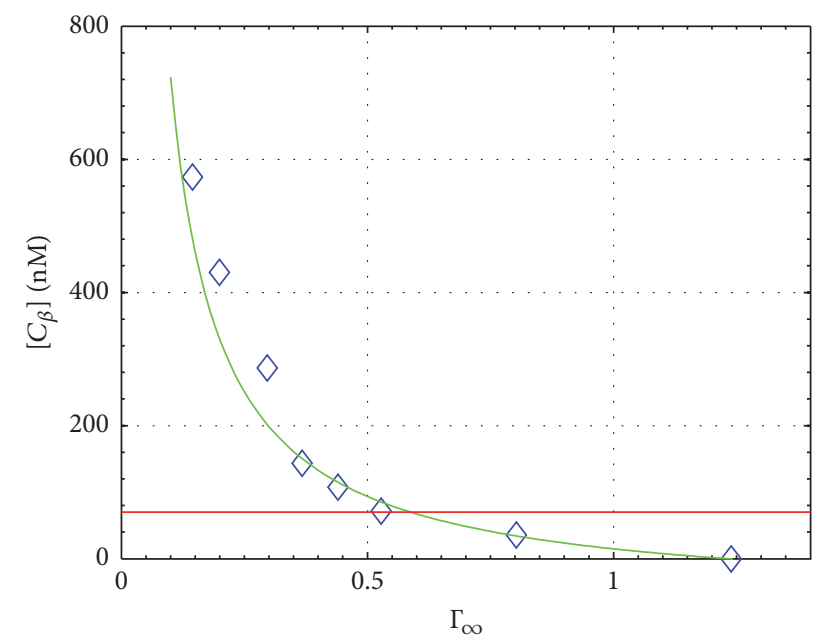

FIGURE 10: $\Gamma_{\infty}$ values $(\diamond)$ obtained from the analysis of the standards reported in Table 3 and calibration function (green line). The constants of the calibration function are $a=99.46(\mathrm{nM})$ and $b=95.27$ (nM) (Pearson coefficient of the fitting $R^{2}=0.981$ ). The horizontal line (red line) represents the maximal residue limit (MRL) defined by the European Medicines Agency for cloxacillin.

3.4. Verification. As described in Section 2.3, verification has been performed by analyzing two sets of milk samples with known antibiotic concentration, thus comparing the known concentration with results provided by the calibrated system. The obtained results are shown in Figure 11 and the absolute values of the percentage errors are shown in Figure 12.

\section{Conclusions}

In this paper, we propose a measurement method and we developed and test a "proof of concept" measuring system that have been demonstrated to be able to detect cloxacillin in milk at concentrations lower than the maximal residue

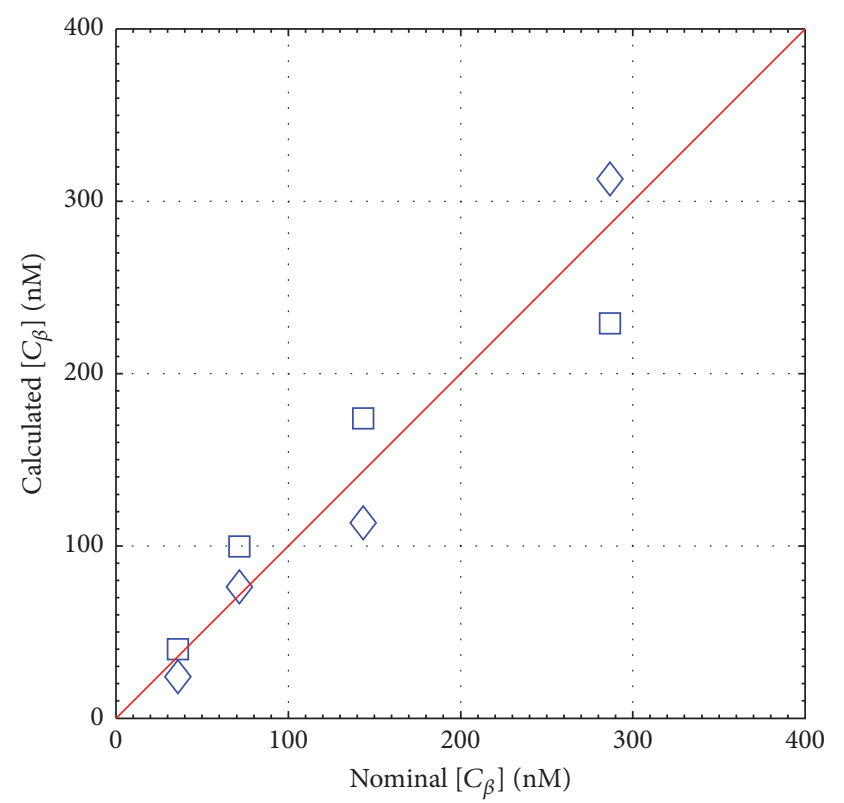

FIGURE 11: Comparison between the nominal antibiotic concentration "Nominal $\left[C_{\beta}\right]$ " and the concentrations estimated by the calibrated system "Calculated $\left[C_{\beta}\right]$ " for the two sets of samples $(\diamond$ and $\square$ ). The diagonal line (red in color version) is the bisector of the first quadrant.

limit (MRL) set by the European Commission. Furthermore, according to the results of the inhibition test reported in Section 3, the method could be suitable for the detection of other $\beta$-lactam antibiotics. However, at the current stage, the method can be used only to verify and quantify the concentration of cloxacillin and the presence of other antibiotics and inhibitors of AmpC can lead to measurement artifacts. Thus, it could be useful for both cattle breeders who want to rapidly test the quality of the milk of animals that have been treated with cloxacillin and inspectors (veterinaries, regulatory agencies, and milk processors) who want to test quickly and with high resolution the presence and concentration of cloxacillin in the milk produced by animals suspected of being treated with such antibiotic, for example, animals suffering mastitis.

Moreover, as discussed in Section 3.3 and Appendix B, the developed measurement method and system are also intrinsically sensitive to the eventual presence of $\beta$-lactamase illegally added to the milk to set to zero the $\beta$-lactams concentration. Therefore, they are potentially able to detect both $\beta$-lactam antibiotics and $\beta$-lactamase enzyme (AmpC) illegally added to milk. Indeed, as discussed in Appendix A and shown in Figure 7, an AmpC (Escherichia coli K12 AmpC $\beta$-lactamase) concentration equal to some $\mu \mathrm{M}$ can be easily detected.

CENTA, the used substrate, is not an expensive cephalosporin that can be prepared from the cephalothin [24]. Also the proposed measuring system is simple, inexpensive, and easy to use and transport to the field.

Much more testing is required and at the present time, there is no guarantee that this method could be applied for the detection of any other $\beta$-lactam antibiotics. Furthermore, 


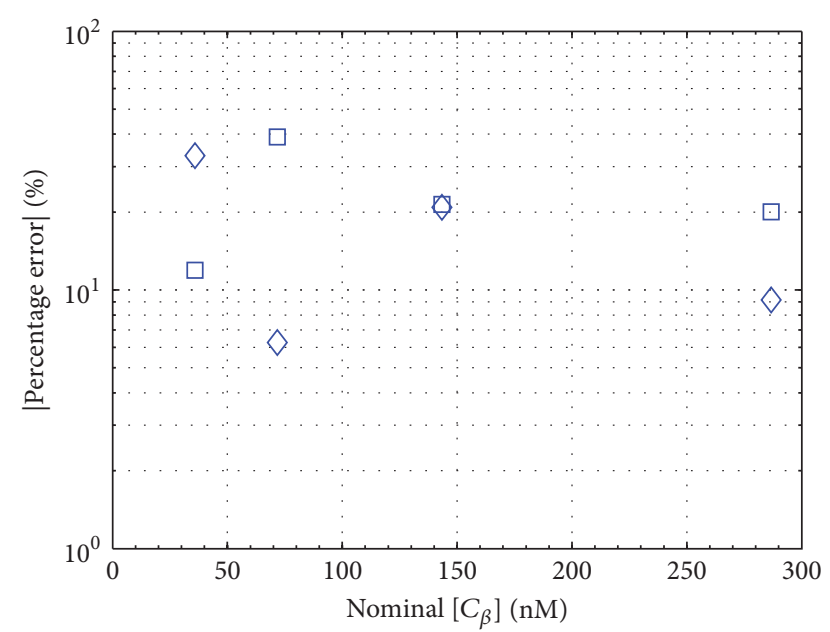

FIGURE 12: Absolute value of the percentage errors for the two sets of samples $(\diamond$ and $\square)$.

the applicability of the method on different milk sample matrices (e.g., goat's and sheep's milk) has not been verified. Moreover, the measure of absorbance around 2.5 and 3.5 is difficult considering the turbidity of milk (as an example, consider the variation of the spectra from Figure 5-buffer-to Figure 6-milk). However, the reported results suggest that the proposed measurement method and measuring system may be able to provide a quantitative measure of $\beta$-lactam antibiotics concentration in milk.

As shown in Figures 7 and 8, although absorbance variation is very small and the values are very high, the proposed measurement method is substantially able to detect cloxacillin at concentration at about $5 \mathrm{nM}$. Indeed, according to (3) and Figure 10, the method is very sensitive at low concentration.

The developed prototype measuring system has been tested and demonstrated at concentration as low as $35 \mathrm{nM}$ (half the MRL concentration set by the European Commission).

The performance of the measuring system can be probably further improved by (i) increasing the signal to noise ratio of the block PD/front-end electronic/ADC (see Figure 9) by redesigning the electronic and/or exploiting best performing components, (ii) tuning the bandwidth of the source, and (iii) exploiting the isosbestic point of the CENTA spectra, thus measuring the absorption of the sample also at $370 \mathrm{~nm}$ (see Figure 5).

Indeed, the used LED has a nominal full width at half maximum at $20 \mathrm{~mA}$ equal to $15 \mathrm{~nm}$; thus, system performance can be reasonably improved by reducing the bandwidth of the source. Such can be accomplished by using optical filters or laser diodes (LD).

Coherent sources such as lasers can lead to problem related to interference (e.g., speckles). However, an automated power control of the laser diode [28] and the analysis of the resulting self-mixing signal can allow us to obtain further information such as the size of milk particles [29], hence potentially providing further information about the milk quality [30].

Moreover, although the absorption peak of the hydrolyzed CENTA is at about $405 \mathrm{~nm}$ (see Figures 5 and 6), as shown in Figure 7, the spectral region around $420 \mathrm{~nm}$ reveals a great variation of absorbance; thus, we are currently investigating the possibility of performing the measure in such spectral region.

Concluding, the proposed measurement method and the measuring system are simple, quantitative, sensitive, and easy to use on the field. Thus, they are useful in delivering rapid results, where access to laboratory analysis is impractical and uneconomic or delays are commercially unacceptable.

\section{Appendix}

\section{A. Derivation of the Measurement Model}

The proposed measurement method is based on the hypothesis that, once the concentration of the AmpC is fixed $\left(\left[E_{0}\right]\right.$ in (1)), the lower the concentration of the $\beta$-lactams antibiotics in the tested milk sample ([I] in (1)), the higher the hydrolyzation of CENTA by the AmpC, and thus the higher the variation of the absorption spectrum of the sample. Hence, the method investigates the concentration of the hydrolyzed CENTA $\left[C_{\mathrm{HC}}\right]$.

According to Beer-Lambert's law, the attenuation of a monochromatic and collimated light beam of irradiance $I_{0}$ traversing a semi-infinite slab of purely absorbing medium composed of $N$ chromophores - the light-absorbing species-is [31]

$$
\begin{aligned}
A(\lambda) & \stackrel{\text { def }}{=} \log _{10}\left[\frac{I_{0}(\lambda)}{I_{T}(\lambda)}\right]=d \cdot \mu_{a}(\lambda) \\
& =d \cdot \sum_{i=1}^{N} \epsilon_{i}(\lambda) \cdot\left[C_{i}\right]
\end{aligned}
$$

where $A$ is the optical absorbance (quantity of dimension one historically referred to as OD (Optical Density)), $I_{T}$ is the irradiance of the beam exiting the slab (transmitted), $\mu_{a}$ is the (overall) absorption coefficient, $d$ is the thickness of the slab (optical path length), $\lambda$ is the wavelength, and $\left[C_{i}\right]$ and $\epsilon_{i}$ are the concentration and the extinction coefficient of the $i$ th-chromophore. To take into account scattering, (A.1) is usually modified as follows [32]:

$$
A(\lambda)=\mu_{a}(\lambda) \cdot d \cdot \mathrm{DPF}+G
$$

where the differential pathlength factor (DPF) takes into account the extended pathlength due to scattering and $G$ represents the losses due to scattering.

In our system, supposing that $A_{\text {CENTA }}(\lambda)$ is the (steadystate) overall optical absorbance of the milk sample after the addition of CENTA-time interval $\left[t_{\mathrm{CENTA}}+\Delta_{t}, t_{\mathrm{AmpC}}\right)$ in Figure 3-from (A.1) and (A.2) the overall optical absorbance 
$A_{\text {AmpC }}$ of the milk sample after the addition of AmpC can be written as follows:

$$
\begin{aligned}
A_{\mathrm{AmpC}}(\lambda, t)= & A_{\mathrm{CENTA}}(\lambda)+\cdots \\
& +\left[C_{\mathrm{HC}}\right](t)\left[\epsilon_{\mathrm{HC}}(\lambda)-\epsilon^{\prime}(\lambda)\right] \cdot d \\
& \cdot \mathrm{DPF}
\end{aligned}
$$

where $\epsilon_{\mathrm{HC}}$ is the extinction coefficient of the hydrolyzed CENTA and $\epsilon^{\prime}$ takes into account the extinction coefficients of the reactants consumed to produce hydrolyzed CENTA.

The digitized output voltage $V_{\mathrm{PD}}(n)$ of the front-end electronic is proportional to the current photogenerated by $\mathrm{PD}$. Hence, omitting noises, the signal $V_{\mathrm{PD}}(n)$ depends on the optical power $P_{T}$ impinging on the photodiode active area:

$$
V_{\mathrm{PD}}=s_{\mathrm{TOT}} \cdot P_{T}+V_{\text {offset }}=k \cdot \frac{P_{0}}{10^{A}}+V_{\text {offset }},
$$

where $s_{\text {TOT }}$ is the product of the sensitivities of the photodiode and front-end electronic, $V_{\text {offset }}$ takes into account the offsets, and $k$ is determined by the sensitivity $s_{\text {TOT }}$ and the geometry of the system. Indeed, the irradiance $I_{0}$ is related to the power $P_{0}$ emitted by the LED and the power $P_{T}$ is related to the irradiance $I_{T}$. Then, to take into account the offset voltage $V_{\text {offset }}$, before starting the measurement, the cuvette was replaced with a beam blocker, thus obtaining

$$
V_{\text {Dark }}=\frac{1}{25} \cdot \sum_{n=1}^{25} V_{\mathrm{PD}}(n) \text {. }
$$

Hence, supposing that $P_{0}, s_{\mathrm{TOT}}$, and the geometry of the measuring system do not change during the time interval $\left[t_{0}, t_{\text {Meas }}\right]$, according to (A.1) and (A.3), the $\Gamma$ function, defined as

$$
\begin{gathered}
\Gamma\left(n-n_{4}\right) \mid n \epsilon\left[n_{4}, n_{\mathrm{TOT}}\right]=\log _{10} \frac{V_{\mathrm{CENTA}}-V_{\text {Dark }}}{V_{\mathrm{PD}}(n)-V_{\text {Dark }}} \\
\approx\left[C_{\mathrm{HC}}\right](t)\left[\epsilon_{\mathrm{HC}}(\lambda)-\epsilon^{\prime}(\lambda)\right] \cdot d \\
\cdot \mathrm{DPF},
\end{gathered}
$$

is proportional to the hydrolyzed CENTA concentration $\left[C_{\mathrm{HC}}\right](t)$. Then, since the final settled hydrolyzed CENTA concentration $\left[C_{\mathrm{HC}}\right](t \rightarrow \infty)$ is determined by the $\beta$-lactams antibiotic concentration $\left[C_{\beta}\right]([I])$, according to (1) the proposed measurement model estimates the final or steadystate pseudo-absorbance $\Gamma_{\infty}$ by interpolating the $\Gamma$ function with

$$
\Gamma(i)=\Gamma_{\infty}-\beta \cdot e^{-\alpha\left(i+i_{x}\right)},
$$

where, as described in Section 2.3, $i$ is the independent variable, $\Gamma(i)$ is the dependent variable, and $\Gamma_{\infty}, \beta, \alpha$, and $i_{x}$ are the parameters.

Note that in our method the CENTA concentration is about three orders of magnitude higher than the AmpC concentration (see Table 3); thus, the substrate concentration in $(1)$ is almost constant $\left([S](t) \approx\left[S_{0}\right]\right)$.
Finally, from (1) the final or steady-state concentration of the hydrolyzed CENTA $\left[C_{\mathrm{HC}}\right]$ can be written as follows:

$$
\left[C_{\mathrm{HC}}\right](t \longrightarrow \infty) \approx \frac{a^{\prime}}{b^{\prime}+\left[C_{\beta}\right]} .
$$

Hence, from (A.6), the antibiotic concentration $\left[C_{\beta}\right]$ can be estimated from the final or steady-state pseudo-absorbance $\Gamma_{\infty}:$

$$
\left[C_{\beta}\right]=\frac{a}{\Gamma_{\infty}}-b
$$

where $a$ and $b$ are the constants of the calibration function that will be obtained experimentally.

Note that, according to (A.9), the proposed method is based on $\Gamma_{\infty}$, thus substantially resulting in an end-point method.

\section{B. $\beta$-Lactamase Detection}

As described in Section 2.2, the proposed measurement method is based on the additions of known amounts of CENTA ( $\left[S_{0}\right]$ in eq. (1)) and, subsequently, AmpC $\left(\left[E_{0}\right]\right)$.

According to eq. (A.1) and as shown in Figure 6, the first addition of CENTA is expected to give rise to a known and fixed variation of the absorbance of the sample under test $\Delta A_{\text {CENTA-0 }}$ (curves $\nabla$ and $O$ ). In other words, according to eq. (A.1):

$$
\begin{aligned}
& A_{\text {CENTA }-0}(\lambda)-A_{\text {Milk }}(\lambda)=\Delta A_{\text {CENTA }-0}(\lambda) \\
& \quad=\left[S_{0}\right] \cdot \epsilon_{C}(\lambda) \cdot d \cdot \mathrm{DPF}
\end{aligned}
$$

where $A_{\text {Milk }}$ is the absorption of the sample before the addition of CENTA, $A_{\text {CENTA-0 }}$ is the expected-no $\beta$-lactamases-absorption of the sample after the addition of CENTA and $\epsilon_{C}$ is the extinction coefficient of the CENTA.

Thus, if at the addition of CENTA some AmpC is already present in the sample, the absorption variation in the time interval $\left[t_{\mathrm{CENTA}}+\Delta t, t_{\mathrm{AmpC}}\right)$,

$$
\Delta A_{\text {CENTA }}(\lambda, t)=A(\lambda, t)-A_{\text {Milk }}(\lambda),
$$

will be different from $\Delta A_{\mathrm{CENTA}_{0}}(\lambda)$ (i.e., curves $\Delta, \triangleright,+, \triangleleft$, $\square, \times, \diamond$ in Figure 6). In particular, according to (A.3), the difference

$$
\begin{aligned}
& \Delta A_{\mathrm{CENTA}}(\lambda, t)-\Delta A_{\mathrm{CENTA}-0}(\lambda) \\
& \quad=\left[C_{\mathrm{HC}}\right](t)\left[\epsilon_{\mathrm{HC}}(\lambda)-\epsilon^{\prime}(\lambda)\right] \cdot d \cdot \mathrm{DPF}
\end{aligned}
$$

is proportional to the concentration of the (eventually) hydrolyzed CENTA $\left[C_{\mathrm{HC}}\right]$ and thus to the $\beta$-lactamase (eventually) illegally added to the milk. Hence, supposing that $\Gamma_{0}$ is

$$
\Gamma_{0}=\log _{10} \frac{V_{\text {Milk }}-V_{\text {Dark }}}{V_{\text {CENTA }}-V_{\text {Dark }}},
$$


recorded during calibration (in the absence of $\beta$-lactamases in milk), in analogy to (A.6),

$$
\begin{aligned}
\Gamma\left(n-n_{2}\right) \mid n & \in\left[n_{2}, n_{3}\right]=\log _{10} \frac{V_{\text {Milk }}-V_{\text {Dark }}}{V_{\mathrm{PD}}(n)-V_{\text {Dark }}}-\Gamma_{0} \\
& \approx\left[C_{\mathrm{HC}}\right](t)\left[\epsilon_{\mathrm{HC}}(\lambda)-\epsilon^{\prime}(\lambda)\right] \cdot d \cdot \mathrm{DPF}
\end{aligned}
$$

is proportional to the concentration of the hydrolyzed CENTA $\left[C_{\mathrm{HC}}\right]$. As a result, the system is able to reveal eventual $\beta$-lactamases illegally added to milk. The higher the concentration of the $\beta$-lactamases illegally added to milk, the higher the variation of the absorption spectra $\Delta A_{\text {CENTA }}(\lambda, t)-\Delta A_{\text {CENTA-0 }}(\lambda)$, resulting in a quantitative method. The achievable detection limit of $\beta$-lactamases reasonably depends on several parameters. However, as shown in Figure 7, few $\mu \mathrm{M}$ of AmpC can be reasonably detected. Indeed, according to Figure 7, the addition of CENTA is expected to produce a negligible variation of the absorption at $420 \mathrm{~nm}$ (from curve $\nabla$, to curve $O$ ). On the contrary, an AmpC (Escherichia coli K12 AmpC $\beta$-lactamase) concentration equal to $13.85 \mu \mathrm{M}$ gives rise to a steady-state variation greater than $0.4 \mathrm{OD}$ (from curve $\nabla$, to curve $\triangle$ ).

Note also that in our method the AmpC concentration is in excess in comparison to the concentration of the $\beta$-lactam; thus, the analysis of $\Gamma\left(n-n_{4}\right) \mid n \in\left[n_{4}, n_{\mathrm{TOT}}\right]$ is expected to be able to reveal the presence of $\beta$-lactams even if some $\beta$-lactamases have been illegally added to milk. Nevertheless, the presence of $\beta$-lactamases illegally added to the milk sample results in a different $\left[E_{0}\right]$ (see (1)); hence, $a$ and $b$ constants of the calibration function are in general no longer valid; thus, the analysis provided by the developed measuring system will be qualitative only.

\section{Conflicts of Interest}

The authors declare that there are no conflicts of interest regarding the publication of this paper.

\section{References}

[1] World Health Organization, WHO Estimates of The Global Burden of Foodborne Diseases: Foodborne Disease Burden Epidemiology Reference Group 2007-2015, WHO Press, 2015, http:// www.who.int/foodsafety/publications/foodborne_disease/fergreport/en/.

[2] J. W. DeVries, J. I. Rader, P. M. Keagy et al., "Microbiological assay-trienzyme procedure for total folates in cereals and cereal foods: Collaborative study," Journal of AOAC International, vol. 88, no. 1, pp. 5-15, 2005.

[3] L. B. Bullerman and A. Bianchini, Food Safety Issues and The Microbiology of Cereals and Cereal Products, John Wiley \& Sons, Inc, 2008, http://dx.doi.org/10.1002/9780470439074.ch15.

[4] P. D. Andrade, R. R. Dantas, T. L. Moura-Alves, and E. D. Caldas, "Determination of multi-mycotoxins in cereals and of total fumonisins in maize products using isotope labeled internal standard and liquid chromatography/tandem mass spectrometry with positive ionization," Journal of Chromatography A, vol. 1490, pp. 138-147, 2017.
[5] C. Zanardi, L. Ferrari, B. Zanfrognini et al., "Development of a sensor system for the determination of sanitary quality of grapes," Sensors (Switzerland), vol. 13, no. 4, pp. 4571-4580, 2013.

[6] G. N. Sagandykova, M. B. Alimzhanova, Y. T. Nurzhanova, and B. Kenessov, "Determination of semi-volatile additives in wines using SPME and GC-MS," Food Chemistry, vol. 220, pp. 162167, 2017.

[7] P. M. Dowling, Antimicrobial Drug Residues in Foods of Animal Origin, John Wiley \& Sons, Inc, 2013, http://dx.doi.org/10.1002/ 9781118675014.ch25.

[8] L. Kantiani, M. Farré, D. Barceló, and D. Barceló, "Analytical methodologies for the detection of $\beta$-lactam antibiotics in milk and feed samples," TrAC Trends in Analytical Chemistry, vol. 28, no. 6, pp. 729-744, 2009.

[9] C. Cháfer-Pericás, Á. Maquieira, and R. Puchades, "Fast screening methods to detect antibiotic residues in food samples," TrAC - Trends in Analytical Chemistry, vol. 29, no. 9, pp. 1038-1049, 2010.

[10] J. Adrian, D. G. Pinacho, B. Granier, J.-M. Diserens, F. SánchezBaeza, and M.-P. Marco, "A multianalyte ELISA for immunochemical screening of sulfonamide, fluoroquinolone and $B$ lactam antibiotics in milk samples using class-selective bioreceptors," Analytical and Bioanalytical Chemistry, vol. 391, no. 5, pp. 1703-1712, 2008.

[11] H. Zhang and S. Wang, "Review on enzyme-linked immunosorbent assays for sulfonamide residues in edible animal products," Journal of Immunological Methods, vol. 350, no. 1-2, pp. 1-13, 2009.

[12] F. Davis and S. P. Higson, "Label-Free Immunochemistry Approach to Detect and Identity Antibiotics in Milk," Pediatric Research, vol. 67, no. 5, pp. 476-480, 2010.

[13] X. Zhang, Y. Zhang, H. Zhao, Y. He, X. Li, and Z. Yuan, "Highly sensitive and selective colorimetric sensing of antibiotics in milk," Analytica Chimica Acta, vol. 778, pp. 63-69, 2013.

[14] G. Merola, E. Martini, M. Tomassetti, and L. Campanella, "Simple and suitable immunosensor for $\beta$-lactam antibiotics analysis in real matrixes: Milk, serum, urine," Journal of Pharmaceutical and Biomedical Analysis, vol. 106, pp. 186-196, 2015.

[15] B. M. Cummins, F. S. Ligler, and G. M. Walker, "Point-of-care diagnostics for niche applications," Biotechnology Advances, vol. 34, no. 3, pp. 161-176, 2016.

[16] M. Beltrán, M. Berruga, A. Molina, R. Althaus, and M. Molina, "Performance of current microbial tests for screening antibiotics in sheep and goat milk," International Dairy Journal, vol. 41, pp. 13-15, 2015.

[17] Y. Chen, Y. Xianyu, J. Wu, W. Zheng, J. Rao, and X. Jiang, "Pointof-Care Detection of $\beta$-Lactamase in Milk with a Universal Fluorogenic Probe," Analytical Chemistry, vol. 88, no. 11, pp. 5605-5609, 2016.

[18] R. J. T. Hoare, "Cloxacillin and veterinary medicine," Australian Veterinary Journal, vol. 53, no. 5, pp. 245-246, 1977.

[19] FAO, "Milk Facts," Food and Agriculture Organization of the United Nations, http://www.fao.org/resources/infographics/infographics-details/en/c/273893/, Tech. Rep., 2015.

[20] A. Zettner, "Principles of Competitive Binding Assays (Saturation Analyses). I. Equilibrium Techniques," Clinical Chemistry, vol. 19, no. 7, pp. 699-705, 1973.

[21] A. Zettner and P. E. Duly, "Principles of Competitive Binding Assays (Saturation Analyses). II. Sequential Saturation," Clinical Chemistry, vol. 20, no. 1, p. 14, 1974. 
[22] S. Schnell and C. Mendoza, "Time-dependent closed form solutions for fully competitive enzyme reactions," Bulletin of Mathematical Biology, vol. 62, no. 2, pp. 321-336, 2000.

[23] C. Bebrone, C. Moali, F. Mahy et al., "CENTA as a chromogenic substrate for studying $\beta$-lactamases," Antimicrobial Agents and Chemotherapy, vol. 45, no. 6, pp. 1868-1871, 2001.

[24] A. Quotadamo, P. Linciano, P. Davoli, D. Tondi, M. P. Costi, and A. Venturelli, "An Improved Synthesis of CENTA, a Chromogenic Substrate for $\beta$-Lactamases," Synlett, vol. 27, no. 17, pp. 2447-2450, 2016.

[25] R. N. Jones, H. W. Wilson, W. J. Novick, A. L. Barry, and C. Thornsberry, "In vitro evaluation of centa, a new betalactamase-susceptible chromogenic cephalosporin reagent," Journal of Clinical Microbiology, vol. 15, no. 5, pp. 954-958, 1982.

[26] K. C. Usher, L. C. Blaszczak, G. S. Weston, B. K. Shoichet, and S. J. Remington, "Three-dimensional structure of AmpC $\beta$ lactamase from Escherichia coli bound to a transition-state analogue: Possible implications for the oxyanion hypothesis and for inhibitor design," Biochemistry, vol. 37, no. 46, pp. 1608216092, 1998.

[27] R. A. Powers, J. Blázquez, G. S. Weston, M.-I. Morosini, F. Baquero, and B. K. Shoichet, "The complexed structure and antimicrobial activity of a non- $\beta$-lactam inhibitor of AmpC $\beta$ lactamase," Protein Science, vol. 8, no. 11, pp. 2330-2337, 1999.

[28] S. Cattini and L. Rovati, "A simple readout electronics for automatic power controlled self-mixing laser diode systems," Review of Scientific Instruments, vol. 79, no. 8, article 084704, 2008.

[29] C. Zakian, M. Dickinson, and T. King, "Particle sizing and flow measurement using self-mixing interferometry with a laser diode," Journal of Optics A: Pure and Applied Optics, vol. 7, no. 6, pp. S445-S452, 2005.

[30] F. Abeni, L. Degano, F. Calza, R. Giangiacomo, and G. Pirlo, "Milk quality and automatic milking: Fat globule size, natural creaming, and lipolysis," Journal of Dairy Science, vol. 88, no. 10, pp. 3519-3529, 2005.

[31] T. Vo-Dinh, Biomedical Photonics Handbook, CRC Press, 2003.

[32] L. F. Ferreira, D. M. Hueber, and T. J. Barstow, "Effects of assuming constant optical scattering on measurements of muscle oxygenation by near-infrared spectroscopy during exercise," Journal of Applied Physiology, vol. 102, no. 1, pp. 358-367, 2007. 


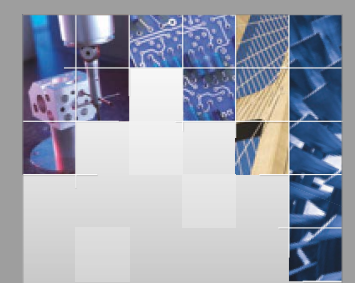

\section{Enfincering}
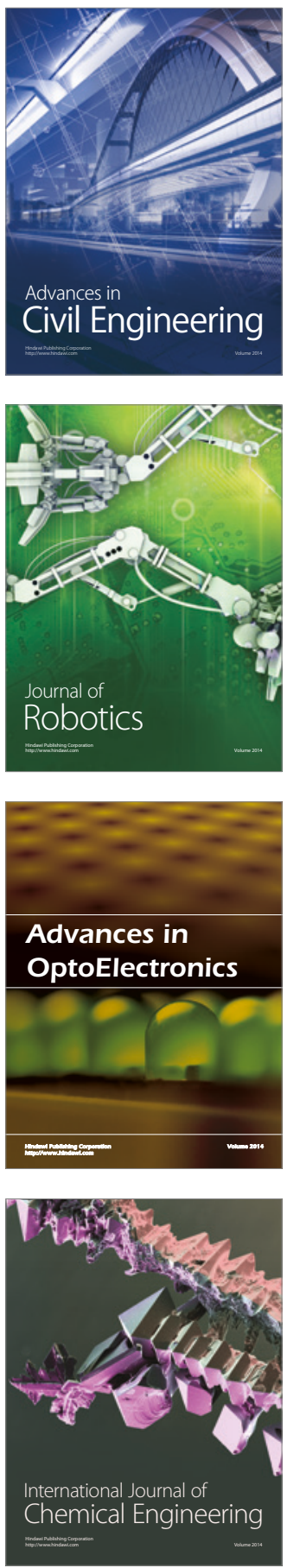

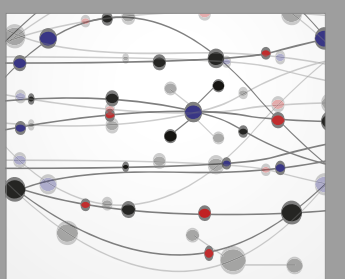

The Scientific World Journal

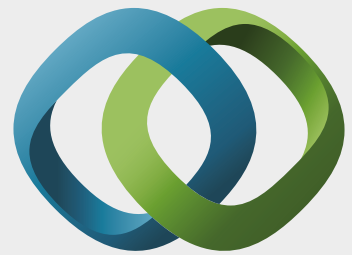

\section{Hindawi}

Submit your manuscripts at

https://www.hindawi.com
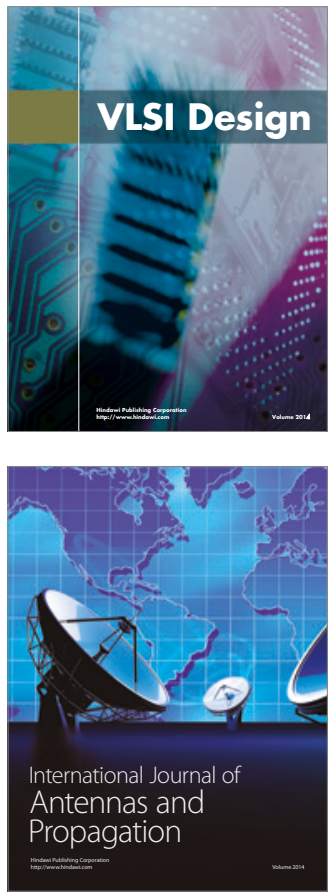

\section{Rotating}

Machinery
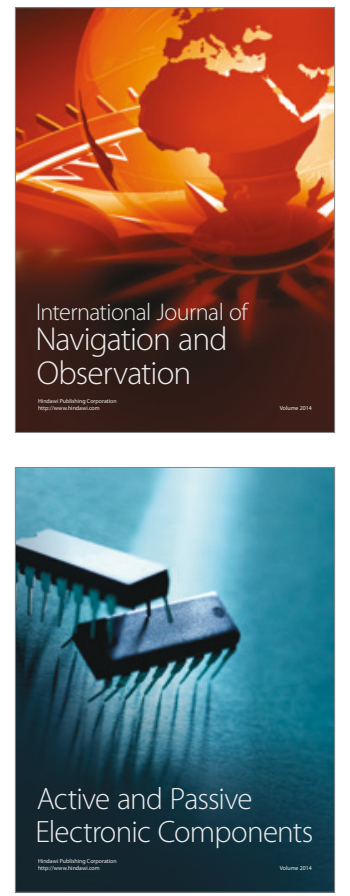
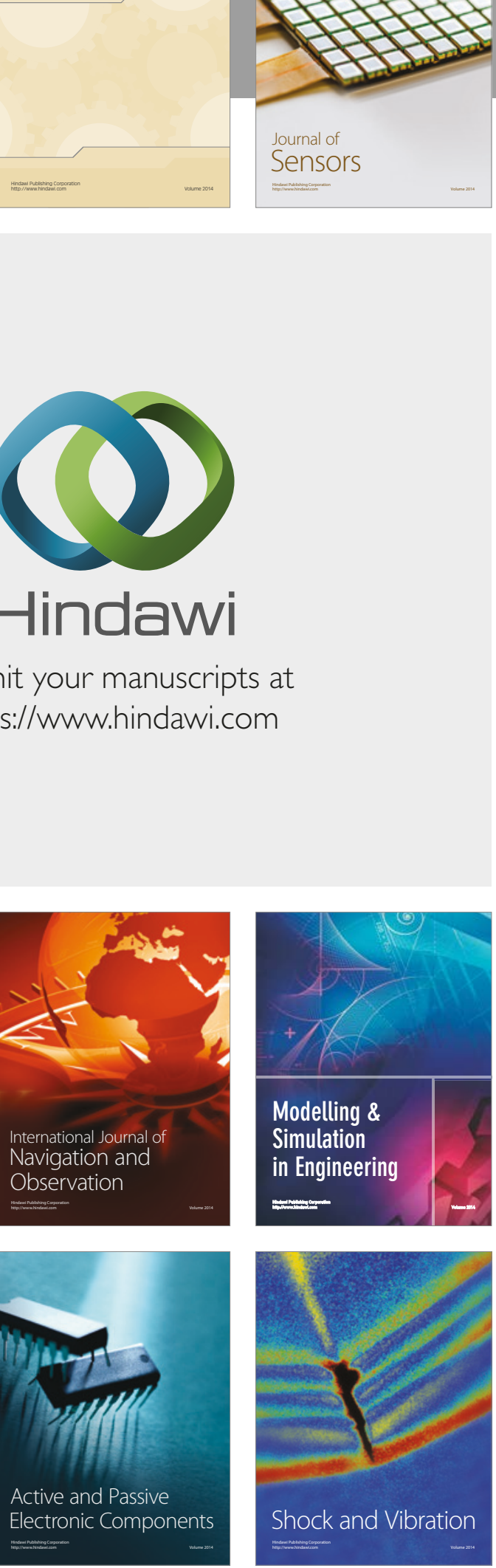
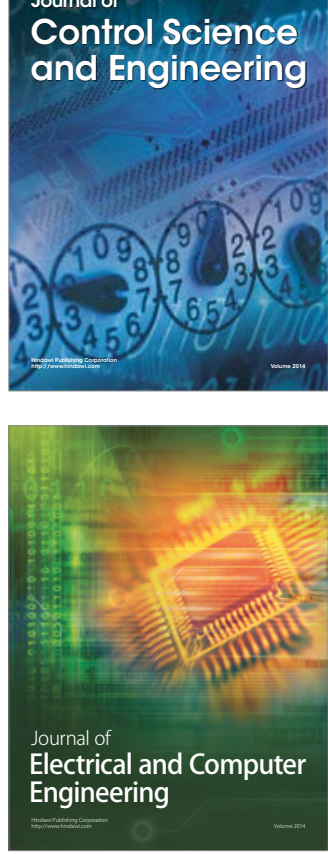

Distributed

Journal of

Control Science

and Engineering
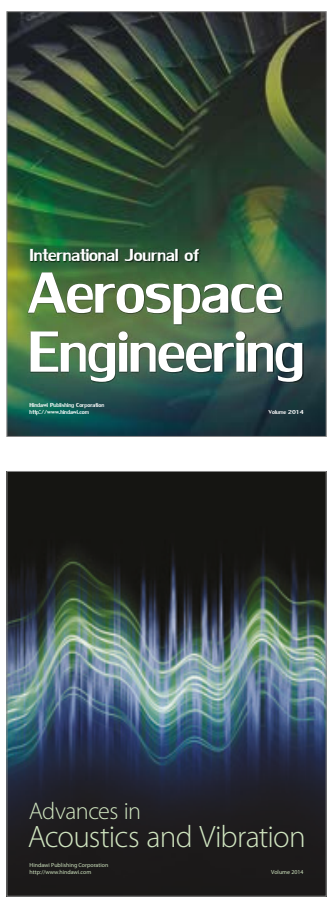

Sensor Networks 